J. RADIAT. RES., SUPPLEMENT 2, 195-201 (1991)

Radiation Carcinogenesis in Mouse Skin and Its Threshold-like Response

HIROSHI TANOOKA AND AKIRA OOTSUYAMA

Radiobiology Division, National Cancer Center Research Institute, Tsukiji, Chuo-ku, Tokyo 104, Japan

(Received December 6, 1990)

\begin{abstract}
Mouse skin/Repeated beta radiation/Tumor/Threshold dose
Experiments on the dose-response of tumor development induced by repeated local beta irradiation of the back of mice are summarized. Results indicated a $100 \%$ tumor incidence on irradiation at $2.5-11.8$ Gy per exposure (dose-saturation effect) and the threshold-like dose response at lower doses. The mechanism underlying these phenomena is discussed on the basis of the tissue-misrepair model, that is misrepair caused by repeated stimulation of cell growth after repeated tissue injury.
\end{abstract}

\title{
INTRODUCTION
}

The carcinogenic effects of single doses of ionizing radiation have been studied extensively, but there have been few studies on the effects of repeated doses of radiation, and especially their dose-response relationship. A fractionated dose of radiation has less carcinogenic effect than a single irradiation with the same total dose (Fig. 1; literatures reviewed in Ref. 1). This reduction in carcinogenicity is supposed to be because the error-free repair mechanism of the cell operates more efficiently at low dose rates than at high dose rates of radiation.

However, fractionation of the dose necessarily involves repetition of treatment, which often increases the carcinogenic effect. A high efficiency of tumor induction in mouse skin is achieved by repeated irradiation with $U V^{2)}$ or soft $X$ rays $^{33}$. The optimum conditions for induction of thymic lymphoma in mice are four repeated sublethal doses of whole body $X$ irradiation $^{4}$. This tumor-enhancing effect of repeated irradiation may involve error-prone repair of repeatedly injured cells or stimulation of error-prone growth of cells in repeatedly injured tissue. The latter mechanism was discussed by Kondo at this symposium ${ }^{5)}$.

These two opposite effects may be involved simultaneously, and their combined effects may result in the unique dose-response relationship of tumor induction described in this report. Here we summarize the results of experiments performed in our laboratory and discuss the mechanism underlying the unique dose-response of the carcinogenic effect of

This work was supported by Grants-in-Aid for Cancer Research from the Ministry of Health and Welfare and the Ministry of Education, Science and Culture of Japan, and a grant from Nissan Science Foundation. 


\section{Beta Radiation}

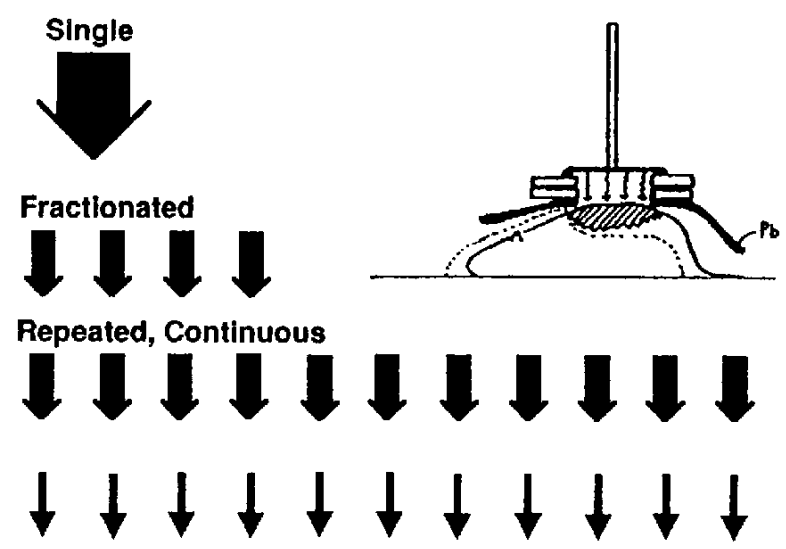

Fig. 1. Diagrams of ${ }^{90} \mathrm{Sr}-{ }^{(2)} \mathrm{Y}$ beta irradiation of the back of mice. Frequency of repeated radiation: 3 times per week.

repeated irradiation.

\section{PERSISTENCE OF LATENT CARCINOGENIC EFFECT OF SINGLE BETA RADIATION}

Single doses of radiation rarely induce tumors in the skin of ICR or C3H/He mice in contrast to rats, in which they induce adnexal tumors at high incidence ${ }^{6,7)}$. We have applied single doses of beta rays, ranging from 5 to $174 \mathrm{~Gy}$, generated from ${ }^{90} \mathrm{Sr}-{ }^{90} \mathrm{Y}$ (maximum beta energy: $2.24 \mathrm{Mev}$; surface dose rate measured by film dosimetry in February, 1985: $2.28 \mathrm{~Gy} / \mathrm{min}$ ) to areas of $2 \mathrm{~cm}$ diameter on the backs of mice, with no success, or a very low efficiency of tumor induction ${ }^{8,9)}$. Single beta irradiation caused unscheduled DNA synthesis (UDS) in cells of the irradiated skin tissue, indicating the occurrence of repair in the irradiated cells ${ }^{10)}$. Application of a chemical promoter, 12-O-tetradecanoylphorbol13-acetate (TPA), after a single dose of 24 Gy was ineffective in producing tumors in our system $^{\text {(I) }}$.

However, application of a chemical carcinogen 4-nitroquinoline 1-oxide (4NQO) at a subcarcinogenic dose (total dose: $2 \mathrm{mg}$ in 20 paintings) after 27 Gy beta irradiation resulted in $13 \%$ incidence of malignant skin tumors. The two alternative orders of treatment (beta rays and then $4 \mathrm{NQO}$, and $4 \mathrm{NQO}$ and then beta rays) resulted in similar yields ${ }^{8)}$. Therefore, the additive effects of these treatments seemed to be due to combination of their two different carcinogenic actions. Prolongation of the time interval between beta irradiation and subsequent treatment with $4 \mathrm{NQO}$ from 11 to $30,63,124,234$, and 408 days did not appreciably change the tumor yield (Fig. 2) ${ }^{9}$. Shubik ${ }^{12)}$ also observed a similar additive effect on application of a promoter, croton oil, an interval of 200 days after beta irradiation. 


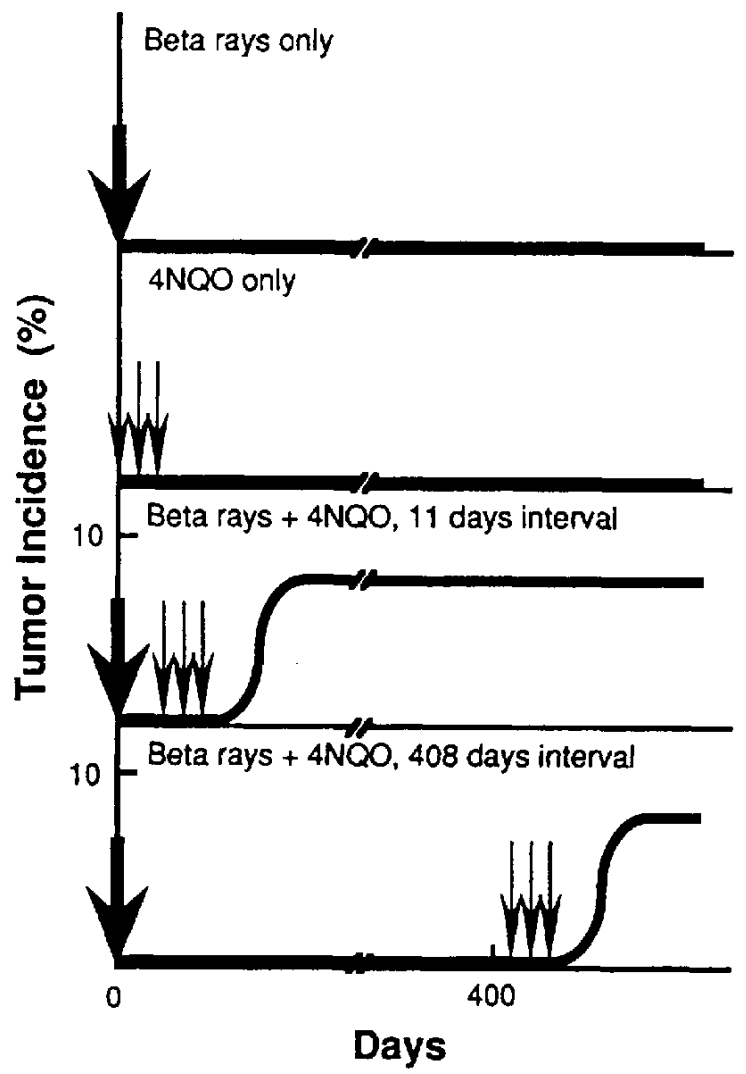

Fig. 2. Schematic figure of increases in skin tumor incidences after single beta irradiation with $2.7 \mathrm{~Gy}, 20$ paintings with $4 \mathrm{NQO}$ (total $2 \mathrm{mg}$ ), and their combination at different intervals. (Summarized from data in Ref, 9)

These results indicate that 1 ) beta irradiation alone is ineffective for inducing tumors, 2) a latent carcinogenic effect persists for a long period without modification in cells surviving irradiation, and 3) this latent effect can be expressed by application of 4 NQO at any time up to at least 408 days after irradiation. A 408 day period represents about half the life span of mice. As epidermal cells are constantly replaced and only stem cells survive in the tissue, this latent effect is thought to be due to mutation or genetic alteration of the stem cells of the skin. Alternatively, the possibility of persistence of physical damage in the immortal DNA strands of stem cells cannot be excluded. 


\section{$100 \%$ TUMOR INCIDENCE BY REPEATED RADIATION (TUMOR- SATURATION EFFECT)}

As single beta irradiation was ineffective for inducing tumors in mouse skin, we next tried to produce tumors by repeating irradiation. For this, the backs of female ICR mice were irradiated with beta rays in the same way as on single-dose irradiation, but treatment was repeated three times a week until the emergence of tumors or death of the mice.

The results are summarized in Figure $3^{1,13)}$. On repeated irradiation of mouse skin with 11.8 Gy per exposure, tumors started to appear from Day 177, and the tumor incidence, calculated by the Kaplan-Meier method ${ }^{14)}$, reached $100 \%$ on Day 477 . The time courses for increase in tumor incidence were similar with lower doses of 2.5, 3.5, and $4.7 \mathrm{~Gy}$ per exposure, indicating a tumor saturation effect in this dose range. In contrast to combined treatment with single beta radiation and $4 \mathrm{NQO}^{8,9)}$ which produced epidermal tumors, repeated beta irradiation produced skin and bone tumors; i.e., squamous cell carcinomas, basal cell carcinomas, fibrosarcomas, and osteosarcomas. That is, tumors developed in both the skin and bone tissues to which the beta rays penetrated.

Furthermore, all the tumors produced by repeating $3 \mathrm{~Gy}$ radiations were monoclonal, as judged from the single phenotypes of phosphoglycerate kinase (PGK-1) of the tumors $^{16)}$ produced in $P g k-1^{a} / P g k-1^{b}$ mice carrying X-chromosome inactivation cellular PGK mosaicism (unpublished data). It is interesting that the tumors produced in repeatedly

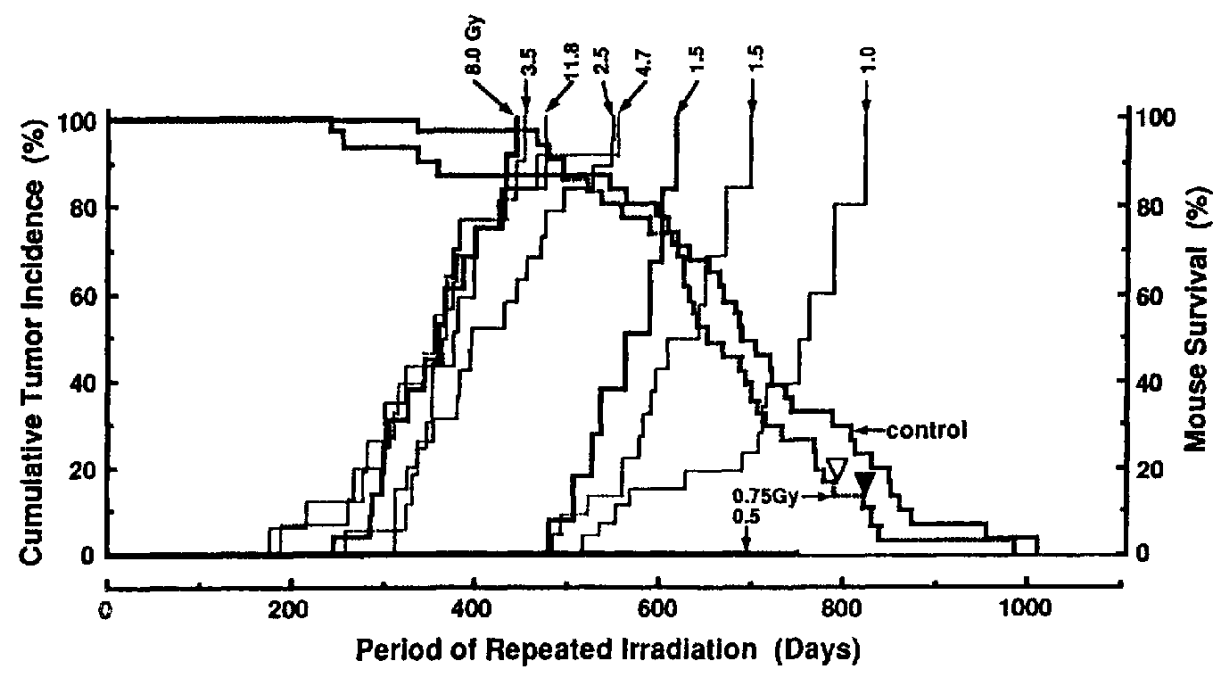

Fig. 3. Effects of repeated beta irradiation. Cumulative incidences of total tumors were calculated by the Kaplan-Meier method and are plotted against the time course of repeated irradiation, together with survival of untreated mice and mice treated with 0.75 Gy per exposure. ( $v$ ) osteosarcoma. ( $\nabla$ ) squamous cell carcinoma. (Data quoted from Refs 1 and 13 with permission from Academic Press, Inc., and unpublished obscrvation) 
irradiated tissue were clones of only single cells, although the tissue was repeatedly injured and many cells must have been recruited, judging from the marked hyperplasia in the skin observed after repeated irradiation.

\section{THRESHOLD DOSE FOR TUMOR INDUCTION BY REPEATED IRRADIATION}

When the dose of radiation per exposure was reduced from $2.5 \mathrm{~Gy}$ to $1.5 \mathrm{~Gy}$, the time before appearance of tumors was abruptly prolonged (Fig. 3) ${ }^{1}$. The first tumor appeared on Day 480 , about 300 days later than the first tumor produced with saturation doses of 2.5-11.8 Gy per exposure. However, the tumor incidence increased with time and reached $100 \%$ by Day 600 . With 1.0 Gy per exposure, further delay in the time of tumor emergence was observed, but again the tumor incidence increased with time to $100 \%$. With $0.75 \mathrm{~Gy}$ per exposure, only two tumors, one squamous cell carcinoma and one osteosarcoma, had developed by nearly the end of the lifespan of $31 \mathrm{mice}^{13)}$. The survival of mice exposed to $0.75 \mathrm{~Gy}$ irradiations was very similar to that of unirradiated control mice (Fig. 3). We are now observing the tumor incidence on exposure to $0.5 \mathrm{~Gy}$ per fraction and have found no tumors among 50 mice until now (Day 700). Thus 0.5 Gy per exposure does not seem to induce tumors. These findings show that on repeated irradiations the dose-response relationship for tumor incidence is not linear.

The dose-response relationship for tumor incidence is seen more clearly from a plot of the time required for $50 \%$ tumor incidence as a function of the radiation dose per exposure (Fig. 4$)^{13)}$. Data on skin and bone tumors were analysed separately, and the points in Fig. 4 show $50 \%$ incidence times of each type of tumor at the indicated doses. It is interesting that all points seem to fit the same curve, which exhibits a sharp rise with radiation doses of below 2 Gy per exposure.

A similar threshold-like response has been reported by Hecker et al. ${ }^{17)}$ for the dose response of tumor incidence with a tumor-promotor such as TPA or teleocidin, applied with various doses to mouse skin initiated with 7, 12-dimethylbenz(a)anthracene (DMBA). However, we found no promoting effect of repeated beta irradiation at 4.7 Gy per exposure on mouse skin initiated with $\mathrm{DMBA}^{11)}$, so the action of repeated beta irradiation is different from that of known chemical tumor promoters.

The mechanism underlying the unique dose response of tumor incidence induced by repeated beta irradiation should be considered ${ }^{(x)}$. It is noteworthy that in our experiments only part of the whole body was repeatedly injured, other parts remaining intact. On repeated irradiation, target cells may accumulate genetic alterations (mutation and recombination), and after certain multiple alterations, cells may acquire the potential for uncontrolled growth and finally develop into a monoclonal tumor through selection of a single clone from many clones. The persistence of the latent carcinogenic effect of a single dose of beta irradiation, which was expressed by addition of $4 \mathrm{NQO}$, can be explained by the persistence of genetic alterations or mutated cells. The threshold-like effect of repeated doses of various levels of radiation on the tumor incidence may also be explained by more 


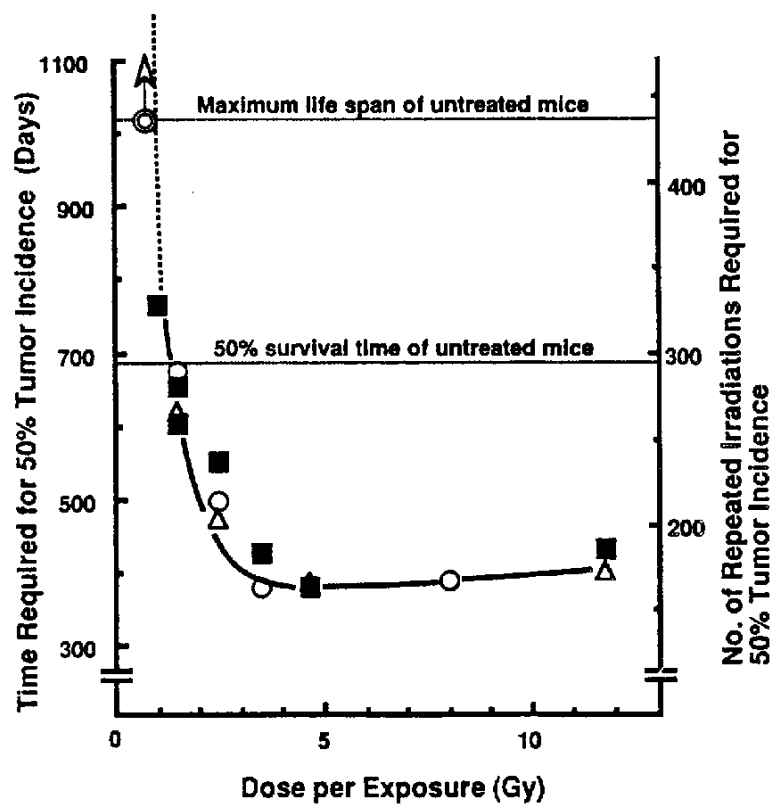

Fig. 4. Thereshold-like response of tumor incidence to repeated beta-irradiation. The time and total number of repeated radiations required for $50 \%$ tumor incidence are plotted against the beta-ray dose per exposure. ( $\square$ ) squamous cell carcinomas and basal cell carcinomas; $(\triangle)$ fibrosarcomas; (O) osteosarcomas; (O) incidences of skin and bone tumors induced by irradiation with $0.75 \mathrm{~Gy}$ per exposure: The incidences did not reach $50 \%$ within the life span of the mice. (Data quotcd from Ref. 13 with permission from Academic Press, Inc.)

efficient error-free repair at low doses than at higher dose of radiation at the cellular level. However, the dose saturation for $100 \%$ tumor incidence can hardly be explained by accumulation of genetic alterations, because the induction of mutations is usually linearly dependent on the radiation dose and a dose-independent event is unlikely to occur at the cellular level.

Therefore, the whole mechanism should be considered at the level of the cell population of the tissue in the whole body. In fact, tissue injuries seem to occur in a thresholdlike manner: depilation of mouse skin did not occur on repeated doses of $0.5 \mathrm{~Gy}$, although the total dose accumulated during 700 days reached $150 \mathrm{~Gy}$, while with repeated doses of 2.5 to $11.8 \mathrm{~Gy}$ per exposurc, depilation and severe hyperplasia occurred in the skin. Uninjured cells in the neighborhood of the repeatedly injured tissue must have received repeated growth stimulation. This injury-stimulated growth may cause misrepair and hence tumors, as suggested by Kondo ${ }^{5)}$. The threshold-like response of tumor incidence on repeated local irradiation seems to be due to the balance of error-free repair operating favorably at low doses of radiation and error-prone repair stimulated by the repeated injury caused by repeated irradiation with a supra-threshold dose. 


\section{REFERENCES}

1. Ootsuyama, A. and Tanooka, H. (1988) One hundred percent tumor induction in mouse skin after repeated $\beta$ irradiation in a limited dose range. Radiat. Res. 115: 488-494.

2. Blum, H.F. (1955) Ultraviolet radiation and cancer. Radiation Biology Vol. 2, pp. 529-559. Hollaender, A. cd. McGraw-Hill, New York.

3. Epstein, J.H. (1972) Examination of the carcinogenic and cocarcinogenic effects of grenz radiation. Cancer Res. 32: 2625-2629.

4. Kaplan, H.S. and Brown, M.B. (1952) Further observations on inhibition of lymphoid tumor development by shielding and partial-body irradiation of mice. J. Natl. Cancer Inst. 12: 427-436.

5. Kondo, S. (1991) Tissue misrepair hypothesis for radiation carcinogenesis. J. Radiat. Res. this volumc.

6. Albert, R.E., Newman, W. and Altshuler, B. (1961) The dose-response relationships of beta rayinduced skin tumors in the rat. Radiat. Res. 15: 410-430.

7. Albert, R.E., Burns, F.J. and Bennctt, P. (1972) Radiation-induced hair-follicle damage and tumor formation in mouse and rat skin. J. Natl. Cancer Inst. 49: 1131-1137.

8. Hoshino, H., Tanooka, H. and Fukuoka, F. (1968) Summation of carcinogenic effect of 4-nitroquinoline 1-oxide and $\beta$-rays. Gann (Jpn. J. Cancer Res.), 59: 43-49.

9. Hoshino, H. and Tanooka, H. (1975) Interval effect of beta-irradiation and subsequent 4-nitroquinoline 1-oxide painting on skin tumor induction in mice. Cancer Res. 35: 3663-3666.

10. Ootsuyama, A. and Tanooka, H. (1986) Unscheduled DNA synthesis after $\beta$-irradiation of mousc skin in situ. Mutation Res. 166: 183-185.

11. Ootsuyama, A. and Tanooka, H. (1987) The tumor-initiating and -promoting effects of ionizing radiations in mouse skin. Jpn. J. Cancer Res. 78: 1203-1206.

12. Shubik, P., Goldfarb, A.R. and Ritchie, A.C. (1953) Latent carcinogenic action of beta-irradiation on mouse epidermis. Nature, 171: 934-935.

13. Ootsuyama, A. and Tanooka, H. (1991) Threshold-like dose of local $\beta$ irradiation repeated throughout the life span of mice for induction of skin and bone tumors. Radiat. Res. 125: 98-101.

14. Hoel, D.G., Walburg, H.E., Jr. and Branch, B. (1972) Statistical analysis of survival experiments. J. Natl. Cancer Inst. 49: 361-372.

15. Ootsuyama, A. and Tanooka, H. (1989) Induction of osteosarcomas in mouse lumbar vertebrae by repeated external $\beta$-irradiation. Cancer Res. 49: 1562-1564.

16. Tanooka, H. (1988) Monoclonal growth of cancer cells: Experimental cvidence. Jpn. J. Cancer Res. 79: $657-665$.

17. Hecker, E. (1990) Threshold doses for tclcocidin and environmental promoters for mouse skin. Proceedings of the 15th International Cancer Congress (Hamburg), \#16.21.01.

18. Tanooka, H. and Ootsuyama, A. (1992) Threshold-like dose response of mouse skin cancer induction for repeated beta irradiation and its relevance to radiation-induced human skin cancer. (Intern. Symp. Skin Carcinogenesis in Man and in Experimental Models, Heidelberg) J. Cancer Res. Clin. Oncol. in press. 